\title{
Liste annexe : ouvrages et articles sur l'internationalisation de l'économie suisse ou sur certains secteurs spécifiques
}

\section{(2) OpenEdition}

\section{Journals}

Édition électronique

URL : http://journals.openedition.org/aspd/1289

DOI : 10.4000/aspd. 1289

ISSN : 1663-9669

Éditeur

Institut de hautes études internationales et du développement

Édition imprimée

Date de publication : 1 décembre 1988

Pagination : 226-230

ISSN : 1660-5934

Référence électronique

"Liste annexe: ouvrages et articles sur l'internationalisation de l'économie suisse ou sur certains secteurs spécifiques », Annuaire suisse de politique de développement [En ligne], 8| 1988, mis en ligne le 13 mars 2013, consulté le 08 septembre 2020. URL : http://journals.openedition.org/aspd/1289 ; DOI https://doi.org/10.4000/aspd.1289 


\section{Liste annexe: ouvrages et articles sur l'internationalisation de l'économie suisse ou sur sur certains secteurs spécifiques}

ACHLEITNER, Paul Michael.- Sozio-politische Strategien multinationaler Unternehmungen: ein Ansatz gezielten Umweltmanagements.- Bern: Haupt, 1985.334p.- (Diss. Hochschule St. Gallen)

liens entre la FT et son environnement socio-économique, politique/ stratégie des FT face aux critiques/ brève étude de cas sur le boycott des produits Nestlé/

ANDREF, Wladimir; MASINI, Jean.- Multinationales et développement. Quelles perspectives? (publication collective)

Revue Tiers Monde.- Paris, PUF, tomme XXIX, janvier 1988.

BLANC, Jean-François.- Suisse- Hong Kong: le défi horloger: innovation technologique et division internationale du travail.- Lausanne: Ed. d'en Bas, 1988.217p.- (coll. Nord-Sud)

internationalisation de la production vers les pays à bas salaires/conditions de travail/ syndicats/

BORNER, Silvio.- «Die Internationalisierung der Industrie»

in: Kyklos, Revue internationale des sciences sociales, 1981, fasc.1, p.14-35

cadre théorique néo-classique n'est pas adapté à la compréhension des causes et effets des activités des FT modernes.

BRAWAND, Antoine.- "Vers une nouvelle division du travail entre le Nord et le Sud?"

in: Information et commentaires, Lyon, Centre Croissance des jeunes Nations, No 58, Janv.- mars 1987, p. 19-31.

BUEHLER, Marcel; LOCHER, Leo.- Geschäfte mit der Armut.- Bornheim Merten: Lamav Taschenbuch, 1984.

les grandes FT pharmaceutiques et les pays en développement/ marché des médicaments/ effets dans les pays en développement/

CAMPBELL, Bonnie K.- Les enjeux de la bauxite: la Guinée face aux multinationales de l'aluminium.- Montréal: Les presses de l'Université de Montréal, 1983.184p.- (Genève, IUHEI)

un chapitre sur les modes d'accumulation internationale du capital et l'industrie mondiale de l'aluminium/

CAVES, Richard.- Multinational enterprise and economic analysis.- Cambridge (Cambridge survey of economic litterature), 1982. 
CHALMIN, Philippe.- Négociants et chargeurs. La saga du négoce international des matières premières.- Paris: Economica, 1985.- 298p. notamment FT Andrél

CLAIRMONTE, F.; CAVANAGH, J.- "Corporate power in selected food commodities". Raw Materiel Reports. Stockholm, vol.1, No3, 1982. pouvoir des FT agro-alimentaire/ dont: André/ Nestlé/ Jacobs-Suchard/

CLAIRMONTE, F; CAVANAGH, J.- Alcool et pouvoir des transnationales.- Lausanne: P.-M. Favre, 1986.- 247p.- (Coll. CETIM)

DAS, Dilip Kumar.- Recent trends in the migration of financial ressources to developing countries and their impact on economic growth. Genève, 1984.- 479p.(Thèse science politique, Genève)

Corée/ Philippines/ Inde/ Analyse théorique de l'impact/

DOBBING, John.- Infant Feeding. Anatomy of a controversy 1973- 1984.- Springer- Verlag, Berlin Heidelberg, 1988.

DUPASQUIER, R.-»Les transferts technologiques internationaux à partir des firmes multinationales: leurs influence sur la balance des paiements"

in: Revue économique et sociale, Bull. de la société d'études économique et sociale, Lausanne, mars 1980, p.17- 22.

causes du transfert de technologie/ effets dans le pays d'origine et le pays d'implantation/

GEORGE, Susan.- Les stratèges de la faim.- Genève: Ed. Grounauer, 1981.365p.- (Coll. Etudes de développement, IUED)

Stratégie des FT et système alimentaire mondial/ Chapitre sur Nestlé/

HAMILTON, Geoffrey.- Les entreprises multinationales. Effets et limites des codes de conduite internationaux.- Paris: PUF, 1984.- 96p.- (Dossiers IRM) Introduction générale à la problématique/ Affaire Nestlé- lait en poudre/

HAUSER, Heinz.- «Joint Ventures. Sonderlösungen für Einzelfälle oder allgemein verwendbare Instrumente der internationalen Kooperation?»

in: Aussenwirtschaft, St. Gallen, Institut für Aussenwirtschafts-, Struktur- und Marktforschung, 1981, p. 176-194.

HEINEN, Hjalmar. Ziele multinationaler Unternehmen. Der Zwang zu Investitionen im Ausland. Wiesbaden: Gabler, 1982.- 313p.- (Uni. Zürich: Diss. Rechtsund Staatswissenschaften)

théories des investissements directs à l'étranger/ motivations des investisseurs/ 
HOEPFLINGER, François.- Das unheimliche Imperium: Wirtschaftsverflechtung in der Schweiz.- Zürich: Eco- Verlag, 1980.- 270p.- (3. vollst. überarb. Aufl.) (l'édition antérieure existe en français)

description des FT suisses et leurs participations/

HUERNI, Bettina.- "Codes of conduct for transnational corporations: an economic analysis".

In : Revue suisse du droit international de la concurrence, Genève, No 14, 1982, p.3-29.

LEVY, Ph.- «Internationale Verhaltenskodizes als Mittel zur Lösung von Konflikten zwischen Staat und multinationalen Unternehmen".

In: Macht und ihre Begrenzung im Kleinstaat Schweiz, Zürich- München: Artemis, 1981, p.179- 190.

LEVY, Ph.- "Investissements directs étrangers dans le Tiers Monde: aide au développement?»

In: Revue économique et sociale, Lausanne, Bull. de la Soc. d'études économique et sociale, août 82, p. 135- 142.

Valeur irremplaçable de l'investissement direct, même si cela n'est pas de l'APD au sens strict/

MERCIAI, Patrizio.- Transfert de technologie: multinationales et pays en développement: arguments et contre-arguments.- Genève: Institut Sandoz d'études en matière de santé et d'économie sociale; Institut universitaire d'études européennes, 1982.- 119p.- (Third World series; 1)

transfert de technologie/ pratiques commerciales restrictives/ brevets/ industrie pharmaceutique/

RIGONI, Raymond; GRIFFITHS, Adrian; LAING, William.- Les multinationales de la pharmacie: polémiques, perceptions et paradoxes.- Genève: IRM; Paris: PUF, 1985.- 123p.- (Dossier de l'Institut de recherche et d'information sur les multinationales; no.5)

RUH, H.- L'éthique économique et les multinationales.- Berne: Institut d'éthique sociale, 1980.

RUH, Hans; PETER, Hans-Balz; HOEHN, Rudolf.- Les banques suisses face à l'éthique sociale.- Berne: Institut d'éthique sociale, 1981.- (2 vol.)

SCHAFFNER, H.- Die Wettbewerbsfähigkeit der schweizerischen Textilindustrie. Diessenhofen: Rüegger Verl., 1982.- (Schweiz. Institut für Aussenwirtschafts-, Struktur- und Marktforschung Hochschule St. Gallen).

indicateurs de compétitivité/ comportement de la concurrence, notamment dans les nouveaux pays industrialisés/ 
SCHNYDER, Klaus: Das multinationale Unternehmen im Spannungsfeld des Gastlandes.- Menschenrechte und nationale Kultur. Mönchengladbacher Gespräche 7.- Anton Rauscher (Hrsg.), Köln 1985.

SCHOOP, Peter.- Finanzwirtschaftliche Probleme und Kontrollinstrumente im multinationalen Konzern.- Diss. Wirtschaftswiss. Zürich, 1983.- 259p.

liens entre la FT et son environnement/ structure interne de la FT et stratégie financière/liens Etats-nation - FT/ sur la base d'entretiens auprès de 15 FT suisses/

SCHWAMM, Henri; MERCIAI, Patrizio.- Les entreprises multinationales et les services.- Genève: Institut de recherche et d'information sur les multinationales, 1985.- 125p.- (Dossiers de l'IRM; No6)

rôle des banques multinationales/ secteur des assurances/

SIEBER, René.- L'analyse des effets de l'investissement direct sur le revenu et sa distribution dans le pays d'accueil.- Berne: $P$. Lang, 1983.- 207p.- (Thèse Fac. des sciences économiques et sociales)

investissement direct comme instrument de l'allocation internationale des ressources générateur de croissance économique/

STRAHM, Rudolf H.- Pourquoi sont-ils si pauvres?.- Lausanne: Braconnière/ Déclaration de Berne, 1986.-220p.

chapitre sur les grandes entreprises et le Tiers Monde/

TINNER, Hanspeter.- Konzernstruktur und Steuerplanung. Strukturierung und Umstrukturierung von schweizerischen internationalen Konzernen aus steuerlicher Sicht.- Bern: Haupt, 1984.- 276p.- (Finanzwirtschaft und Finanzrecht, Bd.39) (Diss. Wirtschaftswiss. St. Gallen)

analyse avec références aux pratiques des FT suisses/ politiques fiscales des Etats et FT/ structure interne de la FT/

WALTER, Herbert.- Standortpolitik multinationaler Banken.- Thun: Verlag Harri Deutsch, 1983.- (Abbildung Wirtschaftswissenschaften, Bd 280)

WEYERMANN, Barbara.- Die Financiers der weissen Herren: eine Untersuchung zum Südafrika-Engagement der Schweizer Banken.- Bern: Aktion Finanzplatz Schweiz- Dritte Welt, 1986.- 59p.

Sources principales pour la bibliographie:

- Bibliographie suisse de statistique et d'économie politique, Berne. Plusieurs années.

- Bibliographie Suisse-Tiers Monde 1980-85, Centre de documentation de 
I'IUED, Institut universitaire d'études de développement, Genève.

- Bibliographie de l'Annuaire Suisse-Tiers Monde 1986 et 1987/88. IUED, Genève.

- Bibliothèque nationale suisse, Berne.

- Centre de documentation i3W, Informationsdienst 3. Welt, Bern.

- Le Livre suisse. Bulletin bibliographique de la bibliothèque nationale suisse, Berne. 